\title{
IMPACT OF PHYSICAL WORKLOAD ON RISK OF INGUINAL HERNIA REPAIR: A DANISH NATIONAL MALE COHORT STUDY
}

Marie Vad, Poul Frost, Morten Bay-Nielsen, Susanne Wulff Svendsen Aarhus Sygehus, Aarhus, Denmark

10.1136/oemed-2011-100382.269

Objectives Repair of inguinal hernia is a common operation. Little is known about aetiological factors, including effects of work-related physical exposures. We wish to evaluate exposure-response relations between daily load lifted, daily frequency of lifting loads weighing $20 \mathrm{~kg}$ or more, and standing/walking for at least $6 \mathrm{~h}$ a day and risk of inguinal hernia operation.

Methods A cohort of all men born between Jan 11938 and Dec 31 1988, still alive and living in Denmark, and having no diagnosis of inguinal hernia before Jan 11998 was established through register linkage between the Danish Civil Registration System and the National Patient Registry. First time events of inguinal hernia repair between Jan 1st 1998 and Dec 312008 were identified in the Danish Hernia Database. Exposure information from a Job Exposure Matrix based on expert judgement (Tine Steen Rubak, PhD thesis 2010) will be allocated to each individual based on Danish ISCO codes for each job held since 1993 obtained from Statistics Denmark. Hazard ratios will be adjusted for age, socioeconomic status, region of residence, and calendar year.

Results The cohort included 1710281 males. 30795 first time lateral and 22556 medial inguinal hernia appeared corresponding to an age standardised incidence of $14.5 / 10000$ person years, 95\% CI $(14.3 ; 14.6)$ and $10.6 / 10000$ person years, 95\% CI $(10.5$; 10.8), respectively.

Conclusions This study will contribute to the knowledge base on risk factors for inguinal hernia repair. Further studies on workload and risk of recurrence and sickness absence after inguinal hernia repair will be conducted. 